\title{
Wide field-of-view volumetric imaging by a mesoscopic oblique plane microscopy with low numerical aperture objectives
}

Wenjun Shao, Ji Yi

Boston University, USA

\section{Abstract Text}

Conventional light sheet fluorescence microscopy (LSFM), or selective plane illumination microscopy (SPIM), enables highresolution 3D imaging over a large volume by using two orthogonally aligned objective lenses to decouple excitation and emission. The recent development of oblique plane microscopy (OPM) simplifies LSFM design with only one single objective lens, by using off-axis excitation and remote focusing [1]. However, most reports on OPM has a limited microscopic field of view (FOV), typically within $1 \times 1$ $\mathrm{mm}^{2}$ [2]. Our goal is to overcome the limitation with a new variant of OPM to achieve mesoscopic FOV. We implemented an optical design of mesoscopic scanning OPM to allow using low numerical aperture (NA) objective lens. The angle of the intermediate image before the remote focusing system was increased by a demagnification under Scheimpflug condition such that the light collecting efficiency in the remote focusing system was significantly improved. We characterized the 3D resolutions by imaging fluorescent microspheres and demonstrated the volumetric imaging on intact whole zebrafish larvae, mouse cortex, and multiple Caenorhabditis elegans (C. elegans). We demonstrate a mesoscopic FOV up to $\sim 6 \times$ $5 \times 0.7 \mathrm{~mm}^{3}$ volumetric imaging, the largest reported FOV by OPM so far. The angle of the intermediate image plane is independent of the magnification. As a result, the system is highly versatile, allowing simple switching between different objective lenses with low (10x, NA 0.3) and median NA (20x, NA 0.5). 


\section{Experiment setup}

The schematic layout of the experimental setup is shown in Fig. 1. OL1 is a low NA objective lens.

\section{Results}

\subsection{Volumetric imaging with $10 \times(0.3 N A)$ configuration}

The resolutions of 10X configuration over the whole FOV of $\sim 6 \times 5 \times 0.6 \mathrm{~mm}^{3}$ were evaluated to be $6.5 \pm 1.1 \mu \mathrm{m}(\mathrm{X}), 7.1 \pm$ $1.2 \mu \mathrm{m}(\mathrm{Y})$, and $34 \pm 6.5 \mu \mathrm{m}(\mathrm{Z})$.

Transgenic zebrafish expressing green fluorescent protein ( $\geq 3 \mathrm{dpf}$ ) were imaged in vivo as shown in Fig. 2 . The size of the acquired volume was $3.2 \times 0.69 \times 0.63 \mathrm{~mm}^{3}$ with a pixel dimension of $995 \times 218 \times 200$.

To demonstrate the extended mesoscopic FOV of the proposed setup, an ex vivo mouse cortex with FITC-infused vasculature was imaged as shown in Fig. 3. The resulting volume size was $5.8 \times 4.9 \times 0.7 \mathrm{~mm}^{3}$ with a pixel dimension of $1800 \times 1550 \times 220$.

\subsection{Volumetric imaging with $20 \mathrm{X}(0.5 \mathrm{NA})$ configuration}

By switching to a 20X objective lens and minor refocusing of the remote focusing system, we can flexibly zoom-in a smaller FOV $\left(\sim 2 \times 2 \times 0.25 \mathrm{~mm}^{3}\right)$ with higher $3 \mathrm{D}$ resolutions $(1.9 \pm 0.6 \mu \mathrm{m}, 2.7 \pm$ $0.5 \mu \mathrm{m}, 11.5 \pm 2.4 \mu \mathrm{m}$, in $x-y-z$ respectively). Figure 4 demonstrated in vivo volumetric imaging of four C.elegans expressing GCaMP6s. 


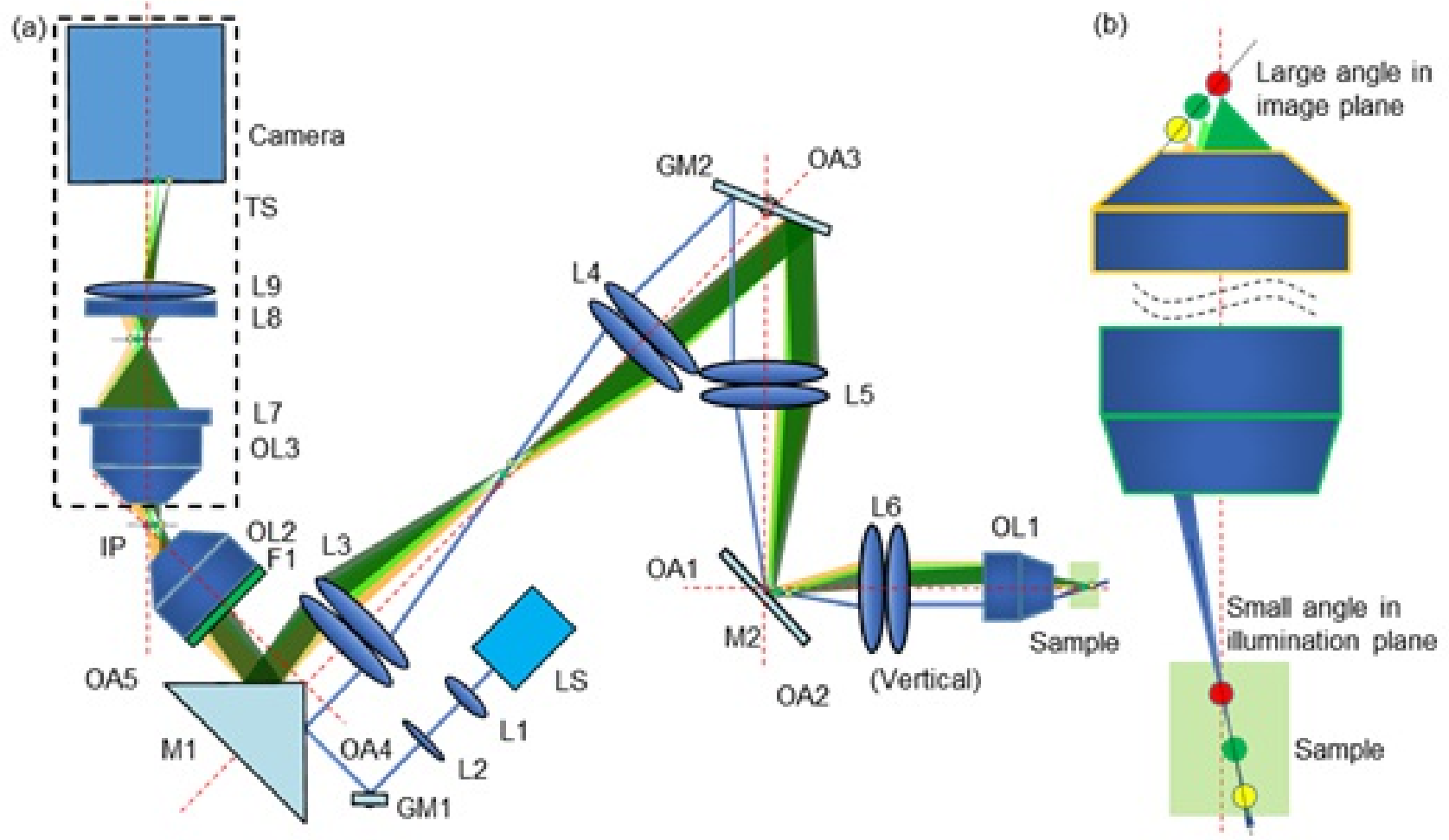

Fig. 1 

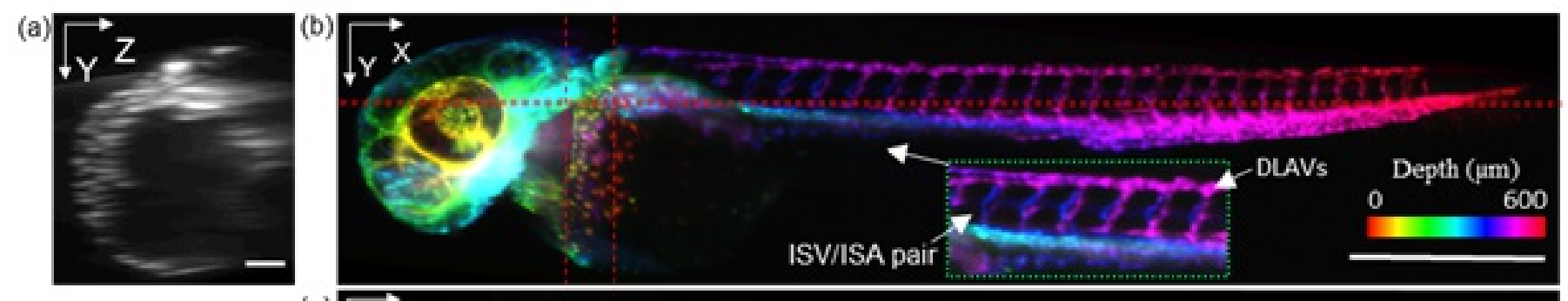

(c) $\vec{z} x$
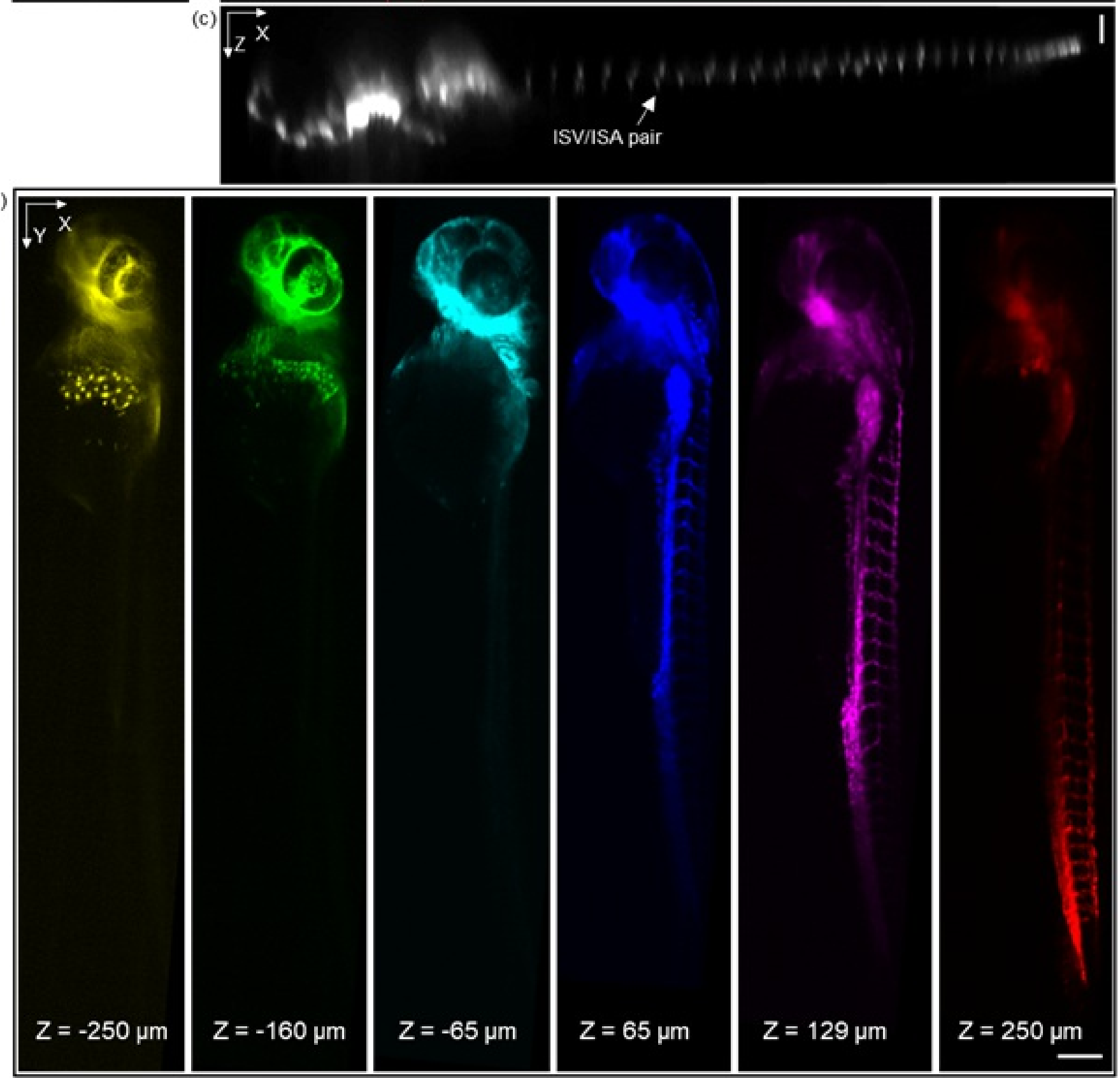

Fig. 2 


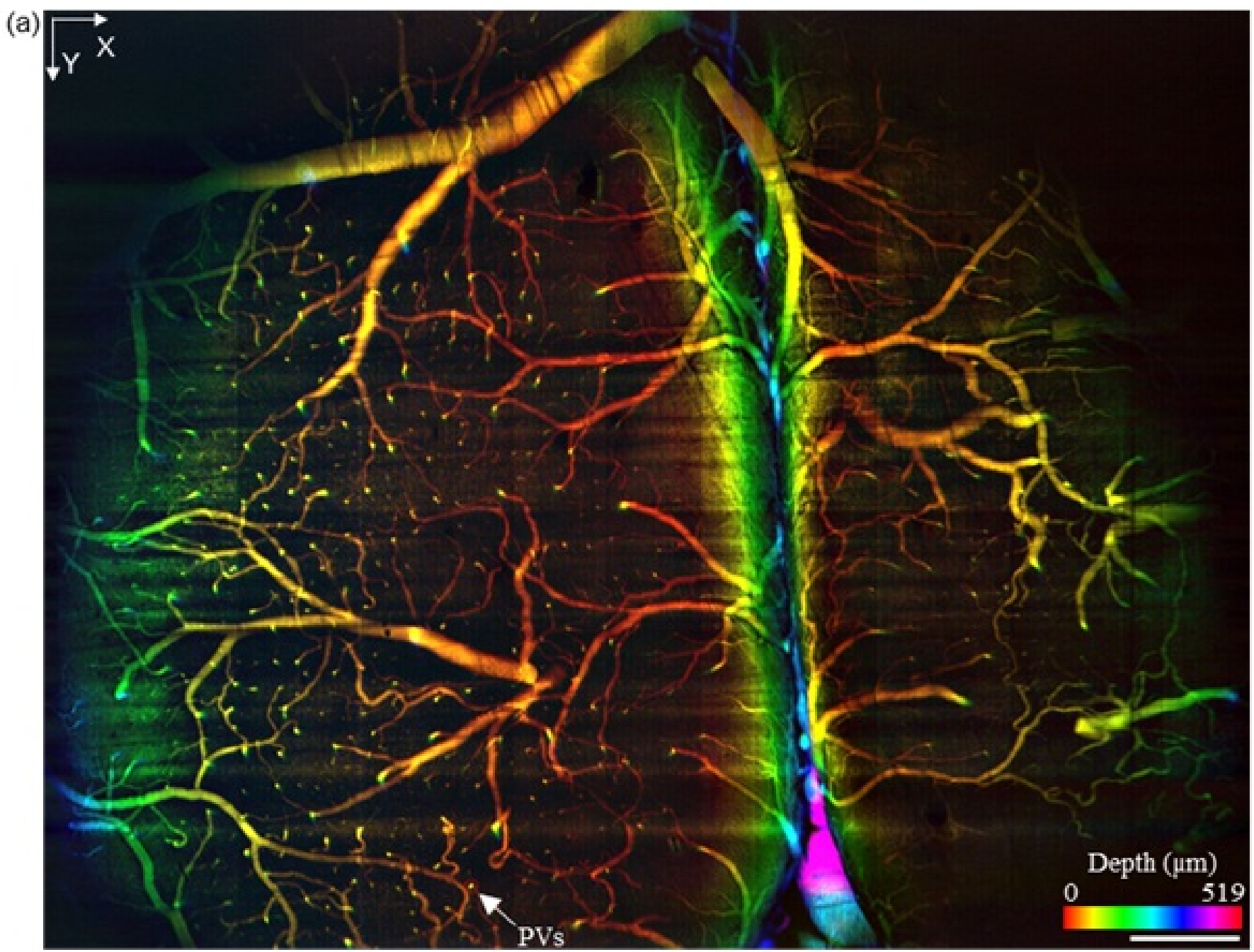

(b)

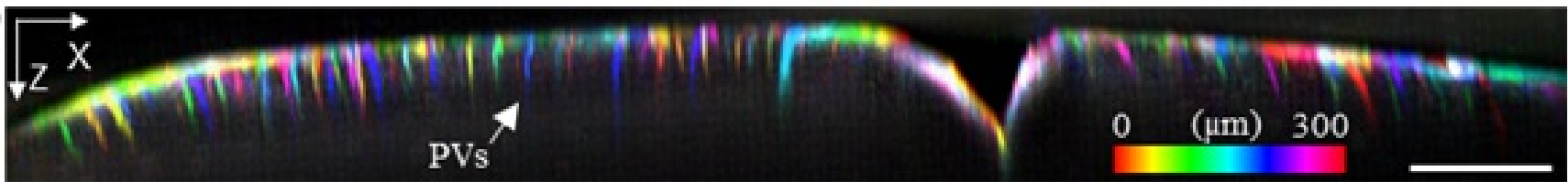

Fig. 3 


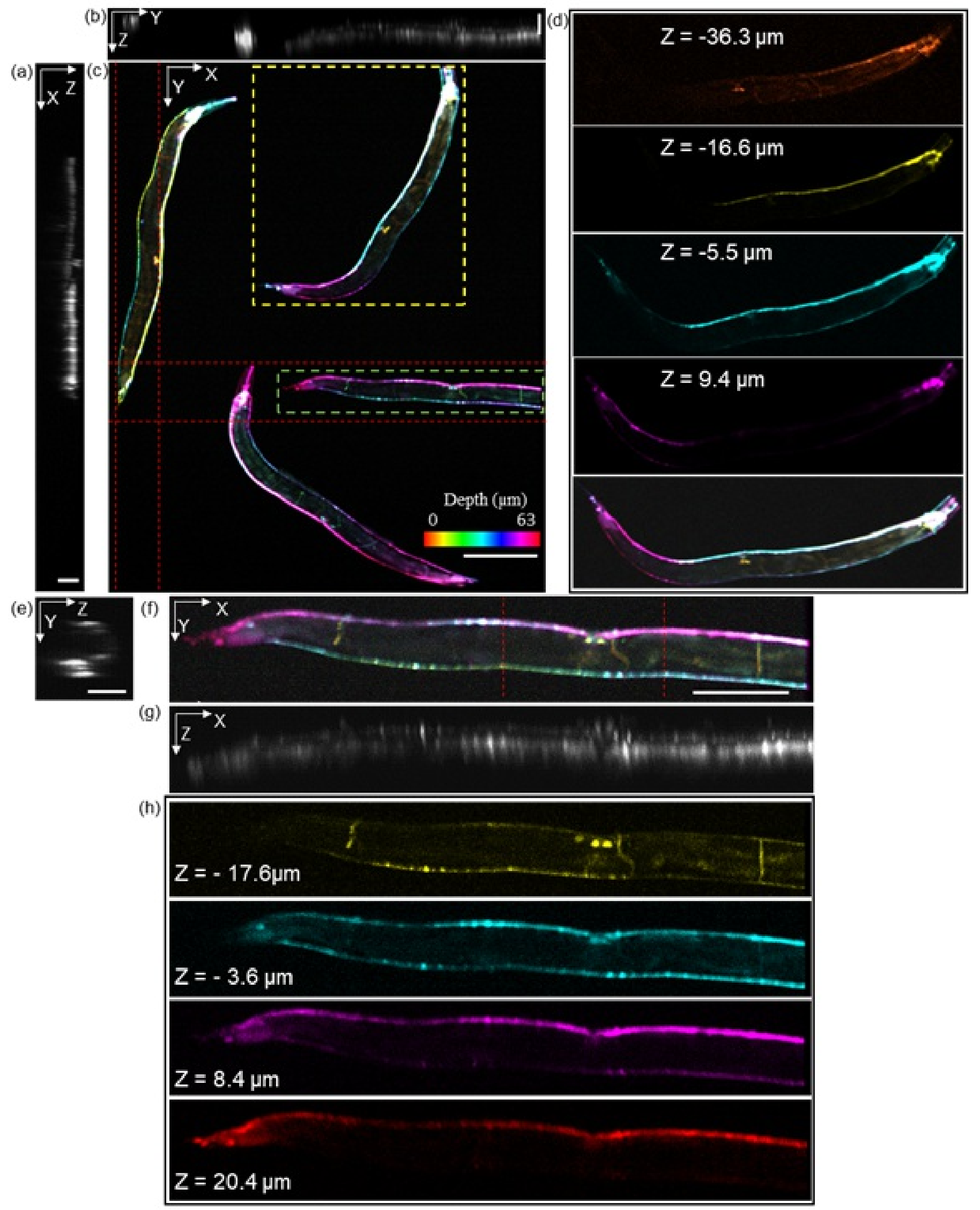

Fig. 4 